\title{
Central fatigue induced by short-lasting finger tapping and isometric tasks: A study of silent periods evoked at spinal and supraspinal levels
}

\author{
P. Arias, V. Robles-García, Y. Corral-Bergantiños, A. Madrid, N. Espinosa, J. Valls- \\ Solé, K.L. Grieve, A. Oliviero, J. Cudeiro
}

\begin{abstract}
The neural substrates of fatigue induced by muscular activity have been addressed in depth in relation to isometric tasks. For these activities, when fatigue develops, it has been noted that the duration of the silent periods (SPs) increases in response to both transcranial magnetic stimulation (TMS) of primary motor cortex or electric cervicomedullary stimulation (CMS). However, fatigue is known to be task-dependent and the mechanisms giving rise to a decrease in motor performance during brief, fast repetitive tasks have been less studied. We hypothesized that fatigue induced by repetitive fast finger tapping may have physiological mechanisms different from those accounting for fatigue during an isometric contraction, even in cases of matched effort durations. In these tasks, we examined the contribution of spinal and supraspinal motor circuits to the production of fatigue. The tapping rate and maximal voluntary contractions (MVC), and TMS- and CMS-evoked SPs were obtained at the time of fatigue, and while subjects maintained maximal muscle activation after fast finger-tapping (or isometric activity) of different durations (10 or $30 \mathrm{~s}$ ). Results showed different mechanisms of fatigue triggered by isometric contraction and repetitive movements, even of short duration. Short-lasting repetitive movements induce fatigue within intracortical inhibitory circuits. They increased TMS-SPs, but not CMS-SPs. On the other hand, isometric contraction had a clear impact on spinal circuits. The consideration of these differences might help to optimize the study of fatigue in physiological conditions and neurological disorders.
\end{abstract}

\begin{abstract}
Abbreviations
$10 \max$-30max, 10 or $30 \mathrm{~s}$ of maximal mode execution; CF, central fatigue; CMAP, compound muscle action potential; CMS, cervicomedullary stimulation; comfort, $30 \mathrm{~s}$ of comfort mode execution; FDI, first dorsal interossesous muscle; $f t$, finger tapping task; iso, isometric task; MVC, (isometric) maximal voluntary contraction; ROM, range of motion; SP, silent period; TMS, transcranial magnetic stimulation
\end{abstract}

Key words

Central fatigue; repetitive movements; human

\section{Introduction}

The finger tapping $(f t)$ test is a reliable procedure used world-wide to evaluate physiological and pathological mechanisms of repetitive movements ( Shimoyama et al., 1990 and Arias et al., 2012). However, when $f t$ is performed at the fastest rate its frequency drops in very few seconds, suggesting the development of muscle fatigue during the task ( Arias et al., 2012 and Teo et al., 2012). Remarkably, muscle fatigue has been chiefly studied when induced by isometric tasks, but the underlying neurophysiological mechanisms of fatigue during fast repetitive movements have been hardly explored.

Muscle fatigue is characterized by a reduction in maximal voluntary contraction (MVC) muscle force (Bigland-Ritchie and Woods, 1984). The progressive failure of muscle-force generating capacity, and failure of impulse conduction through the neuromuscular junction, are known peripheral causes of fatigue (Allen et al., 2008). However, fatigue also involves circuits of the spinal cord, cerebral cortex and subcortical structures (i.e. central fatigue, CF) (Gandevia, 2001), which is of interest in neurological conditions (Zwarts et al., 2008 and Kluger et al., 2013).

In order to evaluate the contribution of supra-spinal and spinal motor circuits to $\mathrm{CF}$, transcranial magnetic stimulation (TMS) permits the study of the motor-evoked potentials resulting from activation of 
M1 and the corticospinal tract. Likewise, electrical cervicomedullary stimulation (CMS) induces motor responses by depolarization of the axons in the corticospinal tract at the cervicomedullary junction (Ugawa et al., 1991).

When a single TMS pulse is applied to the motor cortex during an active contralateral muscle contraction, the electromyographic activity is arrested for a few hundred milliseconds after the motorevoked potential. This period of electromyographic suppression is referred to as a silent period (SP) and can be induced by either TMS or CMS. Therefore, the SP is usually studied while the target muscle maintains a certain level of isometric (iso) contraction. TMS-SP duration increases with stimulus intensity and can reach durations of 200-300 ms in hand muscles ( Cantello et al., 1992 and Inghilleri et al., 1993). However, TMS-SP has two components: the early part corresponds to the first $\approx 75 \mathrm{~ms}$ and is due to spinal inhibition ( Fuhr et al., 1991, Cantello et al., 1992, Inghilleri et al., 1993, Brasil-Neto et al., 1995 and Butler et al., 2012) and involves mechanisms following on from motoneuron excitation, like recurrent (Renshaw) inhibition and axonal after-hyperpolarization. These processes are known to be dependent on the level of preceding motoneuron activity ( Hultborn et al., 1979 and Ziemann et al., 1993).

The second, late part is supraspinal; it is cortical in origin (Fuhr et al., 1991, Cantello et al., 1992, Inghilleri et al., 1993, Ziemann et al., 1993, Brasil-Neto et al., 1995, Butler et al., 2012 and Ziemann, 2013) and is linked to intracortical inhibitory circuits operating via GABAb receptors (Ziemann et al., 1993 and Ziemann, 2013). Thus, it is considered a marker of motor cortical inhibition.

On the other hand, the CMS-SP is induced by spinal mechanisms exclusively (Fuhr et al., 1991, Inghilleri et al., 1993 and Brasil-Neto et al., 1995), sharing the mechanisms of the early part of the TMSSP.

The development of $\mathrm{CF}$ is task-dependent and each task may involve different circuits and mechanisms within the central nervous system (Enoka and Stuart, 1992 and Enoka and Duchateau, 2008). For instance, fatiguing isometric activity (i.e., during a 2 min iso-MVC) increases SP duration in response to TMS and CMS ( Taylor et al., 1996). This suggests that fatigue induced by iso-MVC is generated both at the spinal cord level ( Butler et al., 2003), and, potentially, at a supraspinal level ( Taylor et al., 1996, Di Lazzaro et al., 2003 and Hilty et al., 2011a). Interestingly, repetitive isometric activity has been shown to increase TMS-SP ( Taylor et al., 2000 and Hilty et al., 2011b), and part of this increase seems to be caused by processing of afferent (opioid) signaling from the fatigued muscle to the brain (Hilty et al., 2011b). Conversely, CF induced by non-isometric tasks (1 Hz resisted concentric-eccentric contractions to reaching task-failure) has been attributed to supraspinal mechanisms (Brasil-Neto et al., 1993) after testing the motor-evoked potentials and H-reflex at rest; however a possible contribution of fast-recovery forms of spinal fatigue could not be totally ruled out. This was also the case with CF evaluation after short-lasting and maximal-rate $f t$ ( Arias et al., 2012 and Teo et al., 2012). To avoid this drawback, it is recommended that evaluation of $\mathrm{CF}$ be made during maximal effort, without allowing recovery of the nervous system after the fatiguing activity (Taylor et al., 2000).

In this study, we have evaluated the SPs evoked by TMS and CMS to explore the origin of CF underlying the reduction in motor-output that is expected during a $f t$ task, performed at the fastest possible rate for 10 and $30 \mathrm{~s}$. We compared neurophysiological signs of fatigue induced by $f t$ with those induced by iso-MVC, both of the highest intensity and matched in durations of the effort. In all cases, we evaluated fatigue with no gap in muscle activation to avoid any possibility of recovery of the motor system. Based on the "task-dependency" of fatigue signs ( Enoka and Stuart, 1992, Enoka and Duchateau, 2008 and Enoka et al., 2011), our hypothesis is twofold: Firstly, maximal $f t$ compared to the same duration of maximal iso effort would produce a different reduction of motor output; secondly, changes in excitability of the cortical and spinal circuits under investigation would be different for short-lasting maximal $f t$ and iso tasks.

\section{Experimental procedures}

Experimental protocols complied with the Helsinki declaration and were approved by the University of A Coruña Ethics Committee. Subjects were screened for incompatibility with brain stimulation techniques and were medication-free during the week preceding testing. All subjects consented to participate.

\section{Subjects}

The experiment included two groups of subjects: the TMS-group composed of nine right-handed healthy subjects (eight males and one female; age range 22-38 years), and the CMS-group composed of 
12 right-handed healthy subjects (all males; age range 18-41 years), each group underwent both $f t$ and iso fatigue protocols.

\section{Protocol}

Each subject underwent two experimental sessions, at least one week apart, in randomized order. Sessions were identical but for the type of task executed. In one session, subjects were requested to perform index $f t$, and, in the other session, continuous index finger iso against a force sensor, with the direction of the force "toward" flexion of the first metacarpophalangeal joint of the index finger. Subjects always wore a small goniometer on the index finger metacarpophalangeal joint, and a metal ring at the distal phalanx of the same finger. Subjects pressed or tapped over a thin metal plate placed on the force sensor.

For both $f t$ and iso sessions, subjects executed the tasks in three different modes, and each mode was executed four times (four sets). Therefore, the subjects performed four sets at comfort rate/effort (comfort mode) for $30 \mathrm{~s}$; then four sets of $10 \mathrm{~s}$ at maximal rate/effort (10max mode); and finally four sets of $30 \mathrm{~s}$ at maximal rate/effort (30max mode), always in this order. In all cases there was an inter-set rest period of $1 \mathrm{~min} 40 \mathrm{~s}$.

For the comfort-ft subjects were asked to "tap at their most comfortable rate without feeling fatigued" for as long as the set lasted. In a previous paper ( Arias et al., 2012), we observed that this $f t$ mode is reliable, and performed at a pace of about $1 / 3$ of the maximal rate. Because comfort $f t$ is linked to lower metabolic activity in the sensorimotor cortex compared to faster $(>3 \mathrm{~Hz})$ and slower $(<1 \mathrm{~Hz})$ rates ( Jancke et al., 1998 and Lutz et al., 2005), its use seems to be adequate as a control condition to evaluate the fatigue induced by maximum $f t$. For the comfort-iso participants were asked to press $\approx 1 / 3 \mathrm{MVC}$ with visual feed-back provided by means of online isometric force display. For maximal modes subjects were requested to tap/press as fast/hard as they could from the very beginning to the end of the set.

Fatigue was assessed as either the decrease in frequency or amplitude (for $f t$ ), or in force output (for iso). CF, either supraspinal or spinal, was evaluated by recording the changes in the SP duration in response to TMS or CMS ( Taylor et al., 1996). The stimulation pulses were delivered during brief isometric MVCs (2s-iMVC). The 2s-iMVCs were performed before (pre) and immediately after (with no gap inbetween) task-execution (post), either for $f t$ or iso, for all modes and sets ( Fig. 1). The magnitude of the brief 2s-iMVCs was also an analyzed variable for fatigue monitoring (Bigland-Ritchie and Woods, 1984). An initial session was scheduled to allow some practice and answer all the subjects' questions about the experimental methods (Gandevia, 2001).

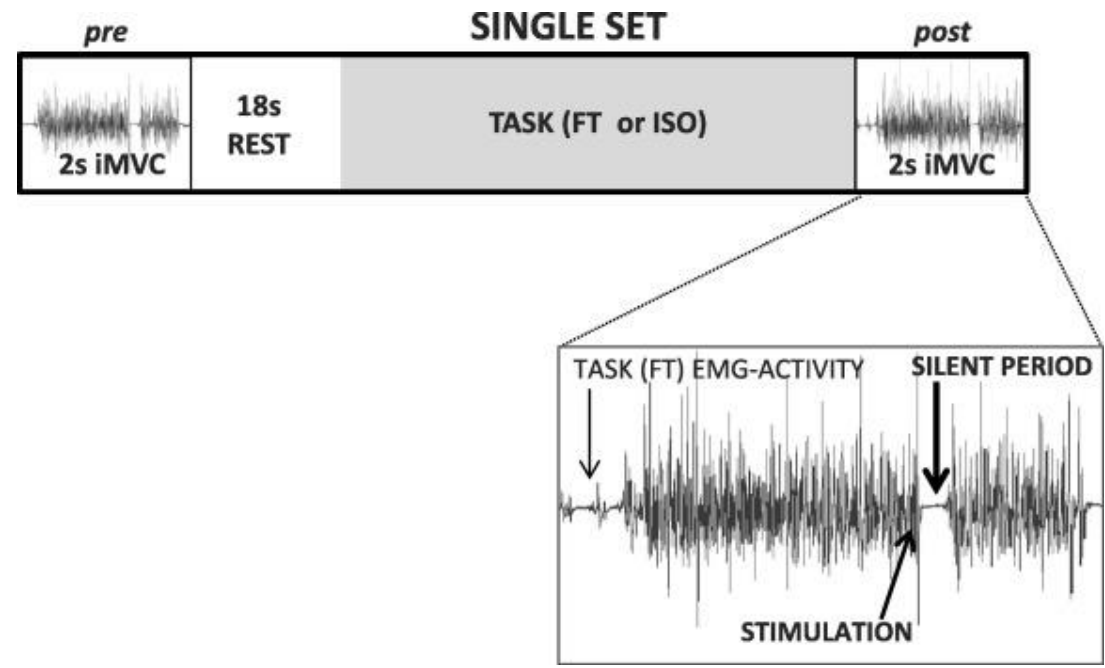

Fig. 1. The set-structure: as soon as the LED was lit subjects performed an isometric maximum voluntary contraction (iMVC) with their index against the dynamometer. The LED off ( $2 \mathrm{~s}$ after) served as a signal to stop the iMVC. During the iMVC (1.5 s after LED on) subjects received an initial test stimulation (pre) (TMS in one group $(n=9)$; electric CMS in the other $(n=12)$. Stimulation induced subsequent silent periods, as shown in the enlarged area. After resting for $18 \mathrm{~s}$, a LED flash indicated the start of the task ( $f t$ in one session; iso in the other). In continuation of the task, and with no resting time, the subjects performed another $2 \mathrm{~s}$-iMVC in response to the LED turning-on, and received stimulation (post; in the same way as pre). 


\section{Setting, recording and stimulation protocols}

The subjects were seated comfortably with the elbow flexed at $90-100^{\circ}$. The forearm, wrist, hand and all fingers except the index were firmly but comfortably fixed to a modified tablet-arm chair, allowing unrestrained degrees of freedom at the metacarpophalangeal joint of the index finger, permitting $f t$. During $f t$, a Biometrics DataLink system (Biometrics Ltd., Gwent, NP11 7HZ, UK) recorded the inter-tap intervals at $0.1 \mathrm{KHz}$ with a thin metal plate and a metal ring, the latter adapted to the distal phalanx of the index finger. It also recorded (at $500 \mathrm{~Hz}$ ) the isometric force exerted during iMVC of the index flexion with a Pinch-Dynamometer (P200), which was placed flat and secured over the table, with the thin metal plate used to record tapping attached flat on its top. A single axis finger goniometer (F35) (sampled at $1 \mathrm{kHz}$ ) controlled the flexo-extension movement amplitudes of the index finger metacarpophalangeal joint. Electromyographic activity from the superficial head of the first dorsal interosseous muscle (FDI) was monitored with surface electrodes in a belly-tendon arrangement, and acquired by means of D360 amplifiers (Digitimer, Welwyn Garden City, Herts), amplified (×250-1000) and band-width filtered between 3-3000 Hz.

EMG were sampled at $10 \mathrm{kHz}$ and stored in a computer by means of a CED 1401 mkII Power A-D converter (Cambridge Electronic Design, Cambridge, UK). This device also controlled the on/off state of LED's (indicating the different phases of execution/rest within the sets) and timing of TMS/CMS pulses.

In separate sessions, in six subjects we recorded the level of EMG activation of the FDI and different intrinsic and extrinsic hand muscles during the $f t$ and different iso tasks; i.e., index iMVC, and iMVC of the 3rd, 4th and 5th fingers working together. Also, we recorded the FDI activation during different index-iMVC tasks applying force against immovable resistances in different directions, toward flexion (the same as above), abduction, adduction and extension. This was done using the same setting, hand position and fixation as during fatigue testing; and adapting immovable resistances to permit iMVC in the different planes. For this purpose the subjects executed two 3s-trials/task, with task presentation order randomized. The inter-trial rest period was one minute.

The trial started with the recording of the compound muscle action potential (CMAP) of each muscle, which was acquired by supramaximal electrical stimulation at the Erb's point (1 ms electric pulses; EBNeuro Stimulator, Italy; cathode lateral, anode medial) with the muscle at rest; $10 \mathrm{~s}$ after stimulation the onset of a LED light indicated the start of 3s-task. The root mean square (r.m.s.) of the CMAP was computed, which served as the divisor of r.m.s. activity during task execution.

For $f t$, we computed the r.m.s. within the tapping cycles (lasting about $150 \mathrm{~ms}$, one per trial) with the highest frequency (shortest inter-tap interval). For the iso tasks, we identified the peaks (one per trial) in the force recordings, then the muscle activity was computed in the $150 \mathrm{~ms}$ around the peaks $(75 \mathrm{~ms}$ before/after the corresponding peak in each trial).

\section{$T M S$}

During the sessions of fatigue evaluation, and for TMS-SP generation, a Magstim $200^{2}$ stimulator delivered monophasic wave-form pulses through a $70 \mathrm{~mm}$ figure of eight coil. The coil was positioned (and marked for reference) to induce currents in a posterior-anterior direction, and placed over the hotspot for the FDI muscle of the executing hand.

The intensity was set to evoke a SP duration $\approx 150 \mathrm{~ms}$ during a $2 \mathrm{~s}$-iMVC in the non-fatigued muscle (this produced TMS motor evoked potentials of about 50\% amplitude of the maximal CMAP); TMS intensity was expressed relative to the individual's active motor threshold, defined as the minimum intensity required to evoked five liminal responses (about $200 \mu \mathrm{V}$ ) in 10 consecutive pulses, in the activated muscle (5-10\% MVC) (Rossini et al., 1994).

\section{CMS}

CMS was applied using a Digitimer D180 stimulator connected to a pair of $\mathrm{Ag}-\mathrm{AgCl}$ electrodes. Electrodes were placed behind the mastoid processes with the anode at the right and the cathode at the left. Active motor threshold was defined as described above for TMS (Rossini et al., 1994); then the stimulation intensity for the protocol was set (about $10 \%$ above this threshold) to have a SP of $\approx 70 \mathrm{~ms}$ in the un-fatigued muscle (this produced CMS motor evoked potentials of about 50\% amplitude of the CMAP). To make sure this intensity did not produce current spread to the spinal roots, the CMS motorevoked potential latency was compared to that obtained at threshold intensity, such that the amplitude of the potentials increased in size with voluntary contraction with no or liminal latency shift when stimulated at the higher level (Taylor and Gandevia, 2004).

Baseline unfatigued SP's durations for TMS and CMS setting were $\approx 150$ and 70 ms respectively, (CMS-SP is much shorter than TMS-SP; (Inghilleri et al., 1993). We set these values away from their 
ceiling to allow a potential modulation induced by fatigue. The mean TMS intensity applied was $17.9 \%$ (s.e.m. 1.0) above the AMT. The mean voltage used in the CMS experiments was 543.4V (s.e.m. 27.1).

\section{Data reduction}

The following dependent variables were analyzed:

Level of muscle activation in intrinsic and extrinsic hand muscles: This is expressed as the percentage of the r.m.s. of the CMAP, and defined as 100 (TASK $\left._{r . m . s} / \mathrm{CMAP}_{r . m . s}\right)$.

In the fatigue sessions we studied the following variables:

Motor output during tasks execution: We considered three measures of motor output for task execution: the tapping frequency, angular amplitude for $f t$ and the force applied for iso. For each of the four Sets and three Modes (comfort; 10max and 30max) we considered two time points which were embedded within task execution: the initial $3 \mathrm{~s}$ (pre) and the final $3 \mathrm{~s}$ (post). To make data from $f t$ frequency and iso torque comparable, we expressed the motor output at all evaluation time points as a function of the maximum obtained at any time point for each task and subject.

For normalizing the $f t$ range of motion (ROM) amplitude, we recorded the maximum (active) ROM of the index finger metacarpophalangeal joint for each subject before the protocol. The score served as divisor for the amplitude displayed at all the individual's evaluation time points during the $f t$ task.

MVC (before and following task execution): We evaluated the MVC at the time of stimulation (MVC in the 50-ms period before the stimulation) either for $f t$ and iso task, normalized to the maximum obtained at any evaluation time-point for each task and subject.

SP duration (recorded during the MVC explained just above): The SP duration was defined as the time-lag from the TMS/CMS pulse to the recovery of the EMG activity during the brief-iMVC, and determined visually by an experienced researcher blind to the conditions (in two separated sessions intrarater reliability was checked on 124 random-chosen SPs, 62 each technique, TMS-CMS). Because we evaluated SP for both TMS and CMS, whose durations are not directly comparable (Inghilleri et al., 1993), the SP durations were normalized. For each subject and task ( $f t$ or iso), we took as $100 \%$ the average value from all the evaluation time-points of the four sets executed in the comfort mode, which served as divisor for all the subject's values for all execution modes. Therefore in the figures representing the SP duration, the unit represents its average duration at comfort mode.

\section{Statistical design}

Statistical design for studying FDI activation during the tasks

To study the level of the FDI activation in comparison with other muscles in each task, we first analyzed whether activation of each muscle was different in the two trials executed. After checking normality (one sample KS test) a paired Student $t$-test was used. Since activation in the two trials was never different $(p>0.05)$, we averaged the values to compare the level of activation of the different muscles and tasks using different models of one-factor ANOVA (with repeated-measures). One model evaluated FDI activation compared to opponens pollicis, abductor digiti minimi, extensor digitorum, and flexor digitorum supperficialis. This model was applied independently to the tasks: $f t$, index iso-flexion, and 3-5th fingers iso-flexion. Another one-factor ANOVA model had four levels, and it was used to compare the activation of the FDI during iso in the four planes; "toward flexion" vs. "extension", "abduction" and "adduction".

\section{Statistical design for studying intra-rater reliability in determining SP durations}

To study intra-rater reliability during SP duration determination (session 1 vs. 2, on the same randomchosen SPs) the intra-class correlation coefficient, and its $95 \%$ confidence interval (95\% CI) were evaluated. For TMS-SP we obtained an ICC $=0.97(0.94-0.98 ; 95 \% \mathrm{CI})$; and for CMS-SP the ICC $=0.97$ (0.96-0.98; 95\% CI).

\section{Statistical design for studying behavior during fatigue tasks}

To study fatigue various repeated measures ANOVA's models were used, after checking the normality of distributions.

For the variables Motor Output (tapping rate-ft; or force-iso) decrement, SP duration, and MVC before and after task execution, we used an ANOVA with repeated measures. The ANOVA included one between-subject factor Group with two levels (the TMS-group and the CMS-group) and several withinsubject factors. In the specific case of the SP, if the factor Group interacted significantly with any of the 
within-subject factors, it means that there was a significant different effect of within-subject factors on the response to spinal or corticospinal stimulation.

The within subject factors were Task ( $f t$, iso), execution Mode (comfort, 10max, 30max), Set (the four sets for each execution mode), and evaluation Time points (pre, post). The levels of the latter factor were termed $i 3$ and $f 3$ when the variable analyzed was the motor output decrease in $f t$ or iso tasks (as it included the initial and final $3 \mathrm{~s}$ embedded in task execution).

For the ANOVA of the ROM amplitude, only analyzed for $f t$, factor Task was excluded.

Results are expressed as the mean and the standard error of the mean (s.e.m). During ANOVA execution the degrees of freedom were corrected with Greenhouse Coefficients $(\varepsilon)$, if sphericity could not be assumed. Significance was set at $p<0.05$. A Bonferroni correction was used for follow-up post hoc comparisons involving multiple levels within the factor.

\section{Results}

The Table 1 indicates the level of activation of the FDI obtained in $f t$ and iso tasks in all subjects ( Fig. 2 shows activations in a representative participant). FDI had a main role in the tasks used in the fatigue protocols (index $f t$ or $i s o$ ). Table 2 shows the normalizing values equivalent to the unit in graphs during the fatigue testing sessions.

Table 1. First dorsal interosseous activation in the tasks of the fatigue protocol (bold fonts) compared to other tasks and muscles

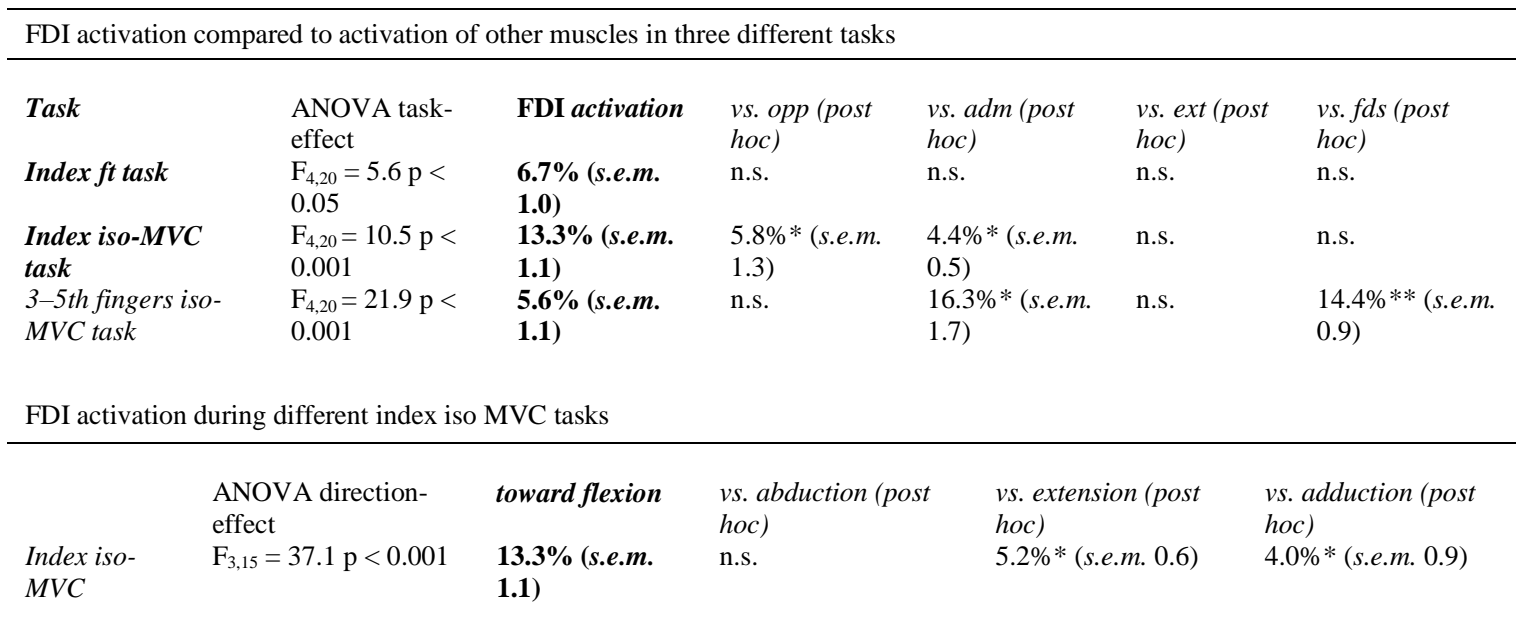

Units $(\%)=100$ (r.m.s. TASK $_{\text {r.m.s. }}$ CMAP). opp: oponens pollicis; adm: abductor digiti minimi; ext: extensor digitorum; fds: flexor digitorum supperficialis. Asterisks indicate Bonferroni post hoc after ANOVA evaluation of main effects. n.s.: not significant. $* p<0.05$.

$* * p<0.01$ 


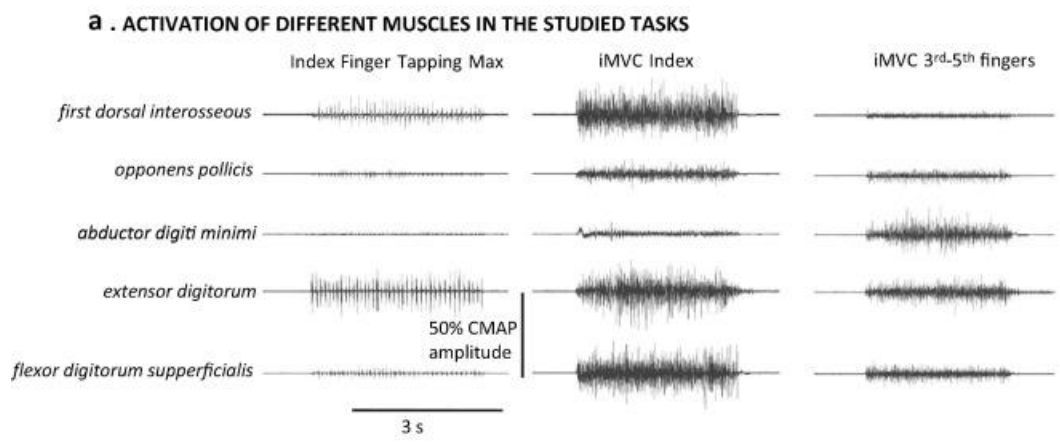

b . ACTIVATION OF THE FIRST DORSAL INTEROSSEOUS MUSCLE IN DIFFERENT MVC TASKS
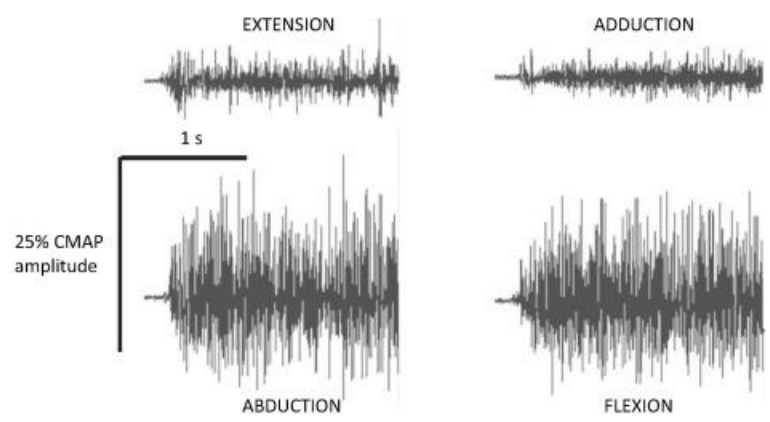

Fig. 2. (a) Activation of the first dorsal interosseous in the ft and iso tasks, for comparison with another task (iMVC flexion of the 3rd, 4th and 5th fingers). Example of individual recordings in one subject (on a separate session) with the settings as describe in Fig. 1. Execution of $f t$ at maximal rate (left recordings); iso MVC with the index finger, as in the protocols (central recordings); and iso MVC flexion with 3-5th fingers together with no index finger pressing (right recordings). The EMG-recordings of the first dorsal interosseous, opponens pollicis, abductor digiti minimi, extensor digitorum and flexor digitorum superficialis are shown in the three tasks. The EMG-amplitudes are scaled to the vertical bar to allow raw-data representation, showing the $50 \%$ of each muscle CMAP amplitude; statistical comparisons were applied for r.m.s. activation; the involvement of the FDI compared to the rest of the muscles is clear and more specific in the task evaluated. (b) Similar maximal EMG-recruitment of the FDI during index finger flexion and abduction, larger than during adduction and extension, all iMVC against unmovable loads.

Table 2. Mean score including all subjects. The score is the $y$-axis unit in graphs

\begin{tabular}{|c|c|c|c|c|c|c|}
\hline Task & $\begin{array}{l}\text { *Max output in task at } \\
\text { any evaluation time- } \\
\text { point }\end{array}$ & $\begin{array}{l}* \text { Max } \\
\text { active full } \\
\operatorname{ROM}^{1}\end{array}$ & $\begin{array}{l}\text { Mean }\left(\%^{1}\right) f t \\
\text { amplitude along } \\
\text { the task }\end{array}$ & $\begin{array}{l}\text { *MVC at any } \\
\text { stimulation time- } \\
\text { point }\end{array}$ & $\begin{array}{l}\text { Mean TMS-SP } \\
\text { duration at } \\
\text { comfort }\end{array}$ & $\begin{array}{l}\text { Mean CMS-SP } \\
\text { duration at } \\
\text { comfort }\end{array}$ \\
\hline$f t$ & $7.1 \mathrm{~Hz}$ (s.e.m. 0.1) & $\begin{array}{l}24.7^{\circ} \\
\text { (s.e.m. 2.0) }\end{array}$ & $28.3 \%$ (s.e.m. 3.9) & $4.7 \mathrm{~kg}$ (s.e.m. 0.3) & $\begin{array}{l}145.1 \mathrm{~ms} \text { (s.e.m. } \\
4.1)\end{array}$ & $\begin{array}{l}67.0 \mathrm{~ms} \text { (s.e.m. } \\
4.0)\end{array}$ \\
\hline
\end{tabular}

* The score utilizes the maximal value from each subject: it is the mean of the maximal scores including all subjects. n.a.: not applicable.

\section{Frequency/force decrement during execution in ft/iso tasks}

The motor output decrements during the task execution (comparing motor output in the initial and final $3 \mathrm{~s}$ of the tasks) were different for the tasks and execution modes $\left(F_{2,38}=8.6\right.$, $\boldsymbol{p}<\mathbf{0 . 0 1}_{\text {TASK }} \times$ MODE $\times$ TIME. $)$ Therefore, we split the analyses for each kind of task.

For $f t$, the frequency reduced over execution $\left(F_{1,19}=86.4, \boldsymbol{p}<\mathbf{0 . 0 0 1}_{\mathrm{TIME}}\right)$, but differently on the three modes $\left(F_{2,38}=43.7, \boldsymbol{p}<\mathbf{0 . 0 0 1}_{\text {MODE }} \times\right.$ TIME $)$; this effect was not differently observed in the two groups 
(subjects stimulated with TMS or CMS; $F_{2,38}=0.4, \boldsymbol{p}>\mathbf{0 . 0 5}$ MODE $\times$ TIME $\times$ GROUP - note that the execution protocol was the same for the two groups of subjects). The frequency of $f t$ at comfort was unchanged, conversely to 10max and 30max (both reduced in the last $3 \mathrm{~s}$ of task execution (i.e., post) compared to the initial $3 \mathrm{~s}$ of task execution (pre), post hoc $\boldsymbol{p}<\mathbf{0 . 0 0 1}$. Set progression had no effect $\left(F_{3,57}=0.3\right.$, $\boldsymbol{p}>\mathbf{0 . 0 5}_{\mathrm{SET}}$, as clearly observed in Fig. $3 \mathrm{a}$, and for this reason the effects are shown pooled at Fig. $3 \mathrm{~b}$.
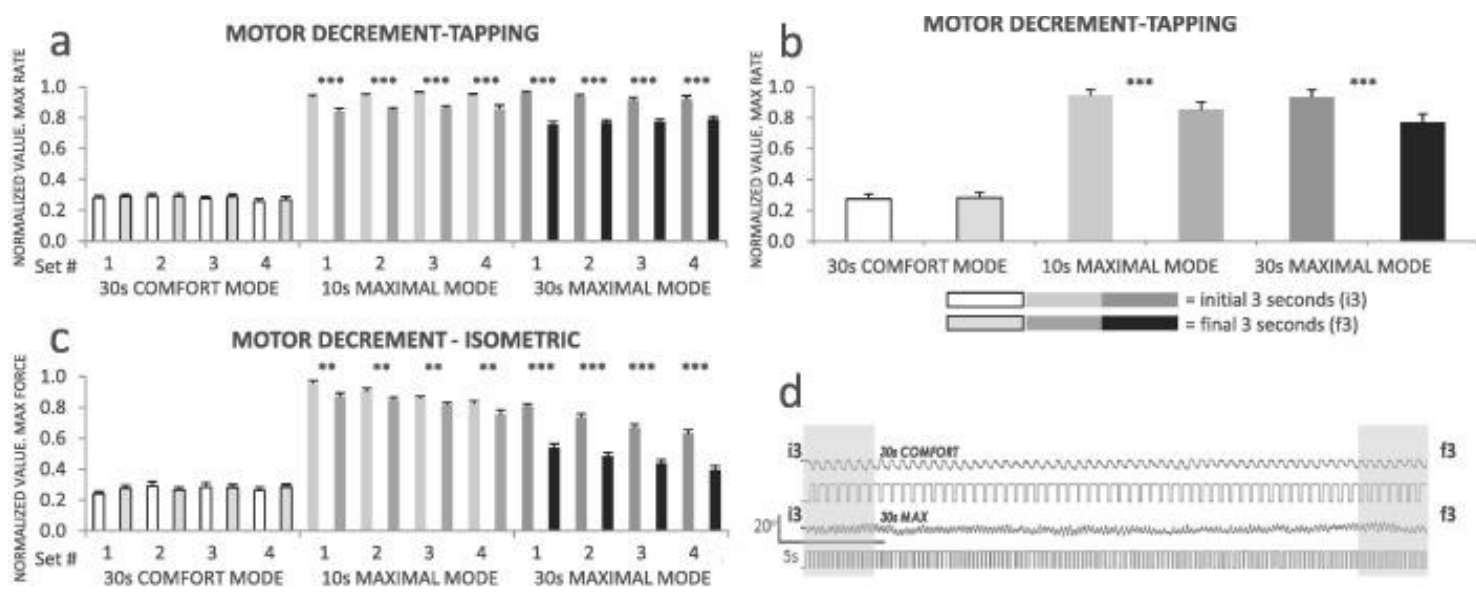

Fig. 3. Motor decrement induced by the tasks. (a) The frequency of $f t$ decreased significantly after the maximal modes; there was no set effect. (b) Same effect seen pooling sets as there was no set effect. (c) Set by set motor decrement along the iso tasks. The force reduced at post after maximal modes and set after set. (d) Representative example in one subject, ft amplitude for comfort and $30 \max$. The amplitude never decreased during the tasks. The unit in the $y$-axis represents the normalized value with respect to the maximal motor output (maximal $f t$ rate, or maximal iso torque obtained at any evaluation time of the corresponding session). In all figures, light colors are the initial $3 \mathrm{~s}$ of execution (pre), dark colors the final $3 \mathrm{~s}$ (post); asterisks denote statistical significance between pre and post, ${ }^{*} p<0.05 ;{ }^{* *} p<0.01 ;{ }^{* * *} p<0.001$.

Fig. $3 \mathrm{c}$ illustrates the force developed during iso in the initial and final $3 \mathrm{~s}$ of the task in the different modes and sets. It was evident that the force dropped at the end of the task for the different modes $\left(F_{2,38}=109.0_{\varepsilon=0.7} \boldsymbol{p}<\mathbf{0 . 0 0 1}_{\mathrm{MODE}} \times \mathrm{TIME}\right)$; and also there was a set effect for some modes impacting the level of the dropping of force at the end of the iso task $\left(F_{6,114}=11.0, \boldsymbol{p}<\mathbf{0 . 0 0 1}_{\mathrm{MODE}} \times \mathrm{SET}\right)$. Again, the execution protocol was identical for the two groups of subjects, those stimulated with TMS and CMS; likely, this is the reason why the dropping of force was not significantly different for the two groups of subjects $(\boldsymbol{p}>\mathbf{0 . 0 5}$ for MODE $\times$ SET $\times$ GROUP Or MODE $\times$ TIME $\times$ GROUP interactions $)$. Then, we followed-up ANOVAs by modes of execution, this was done to understand the before-mentioned differential effects of the modes of execution on the dropping of force at the end of the iso-tasks. We observed no effects of comfort-iso on force drop at post $(\boldsymbol{p}>\mathbf{0 . 0 5}$ for any main effect or interaction). For 10max-iso, the execution dropped significantly at post $\left(F_{1,19}=13.4, \boldsymbol{p}<\mathbf{0 . 0 1}_{\mathrm{TIME}}\right)$, and set after set $\left(F_{3,57}=13.7\right.$, $\left.\boldsymbol{p}<\mathbf{0 . 0 0 1}_{\mathrm{SET}}\right)$. A similar pattern of force-drop was observed for 30 max-iso $\left(F_{1,19}=174.5, \boldsymbol{p}<\mathbf{0 . 0 0 1}_{\mathrm{TIME}}\right.$ and $F_{3,57}=39.6_{\varepsilon=0.7} p<\mathbf{0 . 0 0 1} 1_{\mathrm{SET}}$ ).

\section{Finger angular amplitude during tapping}

During $f t$, the expressions of fatigue might affect not only the tapping-frequency, but also the tapping amplitude. For this reason, we analyzed the ROM amplitude at the first $(i 3=$ pre $)$ and last $(f 3=$ post $) 3 \mathrm{~s}$ of the task. Remarkably, during $f t$ the ROM amplitude was not significantly different at pre vs. post (i3 vs. $f 3 ; F_{1,19}=1.7, \boldsymbol{p}>\mathbf{0 . 0 5}_{\mathrm{TIME}}$; Table 2 ). Likewise, it is worth mentioning, that in all sets, the mean ROM amplitude was not different for the three tapping modes either $\left(F_{2,38}=2.3_{\varepsilon=0.7} \boldsymbol{p}>\mathbf{0 . 0 5}_{\mathrm{MODE}}\right.$; Fig. $3 \mathrm{~d}$ shows an example of the ROM amplitude in one subject). These effects on motor execution were not differently expressed for the subjects stimulated with TMS or CMS (identical task for both groups). 


\section{MVC at the time of stimulation}

The MVC at the time of stimulation (Fig. 4a) was reduced at post differently for the $f t$ and iso tasks $\left(F_{1,19}=30.1, \boldsymbol{p}<\mathbf{0 . 0 0 1}_{\text {TASK }} \times\right.$ TIME ; Fig. $\left.4 \mathrm{~b}-\mathrm{d}\right)$. We followed by splitting the analyses by task-type.

a

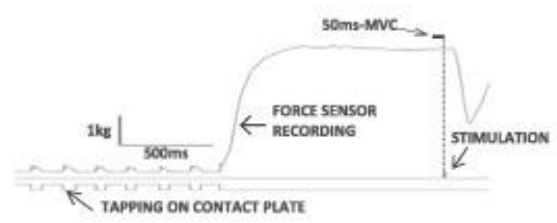

C

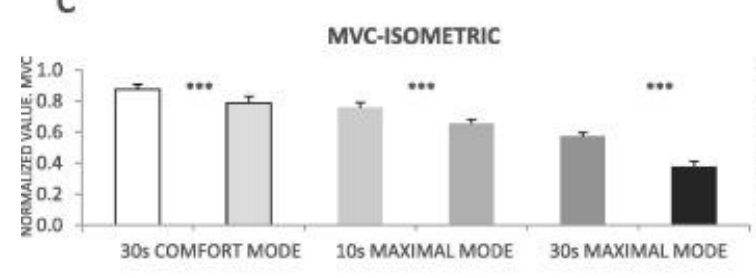

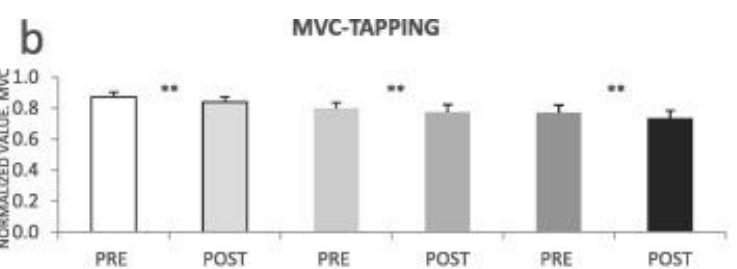

d

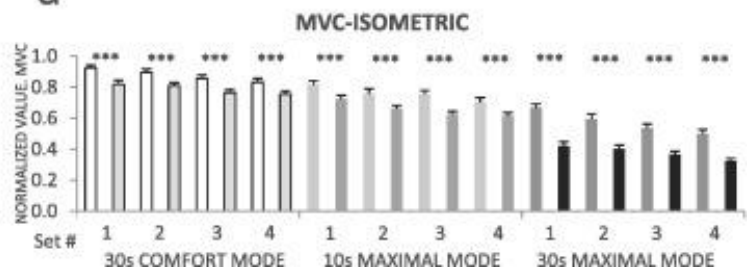

Fig. 4. (a) Example of force recording at post-ft. MVCs shown in 4(b-d) sections are acquired in the 50 ms prior to stimulation. (b) MVC decreased after $f t$ (post) in all modes and from mode to mode. (c-d) MVC decrease after iso (post) in all modes and also from mode to mode and set to set.

For $f t$ tasks, MVC force was reduced at post (i.e., in the MVC performed right at the end of tapping; $\left.F_{1,13}=12.3, \boldsymbol{p}<\mathbf{0 . 0 1}_{\mathrm{TIME}}\right)$ and differed also for the three modes of tapping $\left(F_{2,38}=18.7, \boldsymbol{p}<\mathbf{0 . 0 0 1}_{\mathrm{MODE}}\right)$. Thus, post MVC was weaker after tapping (vs. pre), at all modes and sets ( Fig. 4b), and it also was reduced from mode to mode.

A different matter happened for iso; we initially observed that the MVC decrease at post was different for the three modes $\left(F_{2,38}=15.9, \boldsymbol{p}<\mathbf{0 . 0 0 1}_{\mathrm{TIME}} \times \mathrm{MODE}\right.$; Fig. $\left.4 \mathrm{c}, \mathrm{d}\right)$. Then, we followed-up the analyses by modes. The analyses indicated that for comfort and 10max the MVC dropped at post and set after set (i.e., factors TIME and SET for these to modes were always significant $(\boldsymbol{p}<\mathbf{0 . 0 0 1})$. This was also the case of 30 max, but in addition, the responses at pre and post also differed for the different sets $\left(F_{3,57}=4.9\right.$ $\varepsilon=0.7 p<0.01_{\text {SET }} \times$ TIME $)$ notwithstanding the fact that post hoc pre vs. post were always significant $(\boldsymbol{p}<\mathbf{0 . 0 0 1}$, see Fig. 4c, d).

Again, in agreement with the other variables described thus far, these responses were not significantly different for the subjects stimulated with TMS or CMS (i.e., factor group did not interact significantly in any case), and comparable levels of fatigue were shown in the two groups for each task.

\section{SP adaptation to maximal ft and iso tasks}

As described in the introduction, the SP duration increases with CF. Therefore, we analyzed if SP duration changed after execution (at post); and also if the putative change was different for $f t$ and iso. In one group of subjects the SP was induced by TMS in the two different task-sessions ( $f t$ and iso); in the other it was induced by CMS in the two sessions. Therefore, in all cases, the task-dependency ( $f t$ vs. iso) changes in SP were evaluated for the two kinds of stimulation (i.e., in the two groups). As mentioned above, fatigue did not affect differently the motor execution in the two groups and tasks; thus we compared SP's duration evoked by TMS and CMS in the two tasks; to make their baseline duration comparable the durations were normalized. For this purpose the SP duration at comfort was considered the reference for each kind of stimulation.

The normalized SP durations increased after execution (at post), in a significant different manner for

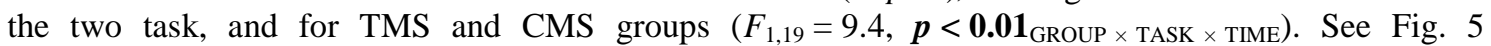
(individual's raw-examples), and Fig. 6. We split the analyses to evaluate the task-dependency effect on the two groups. 

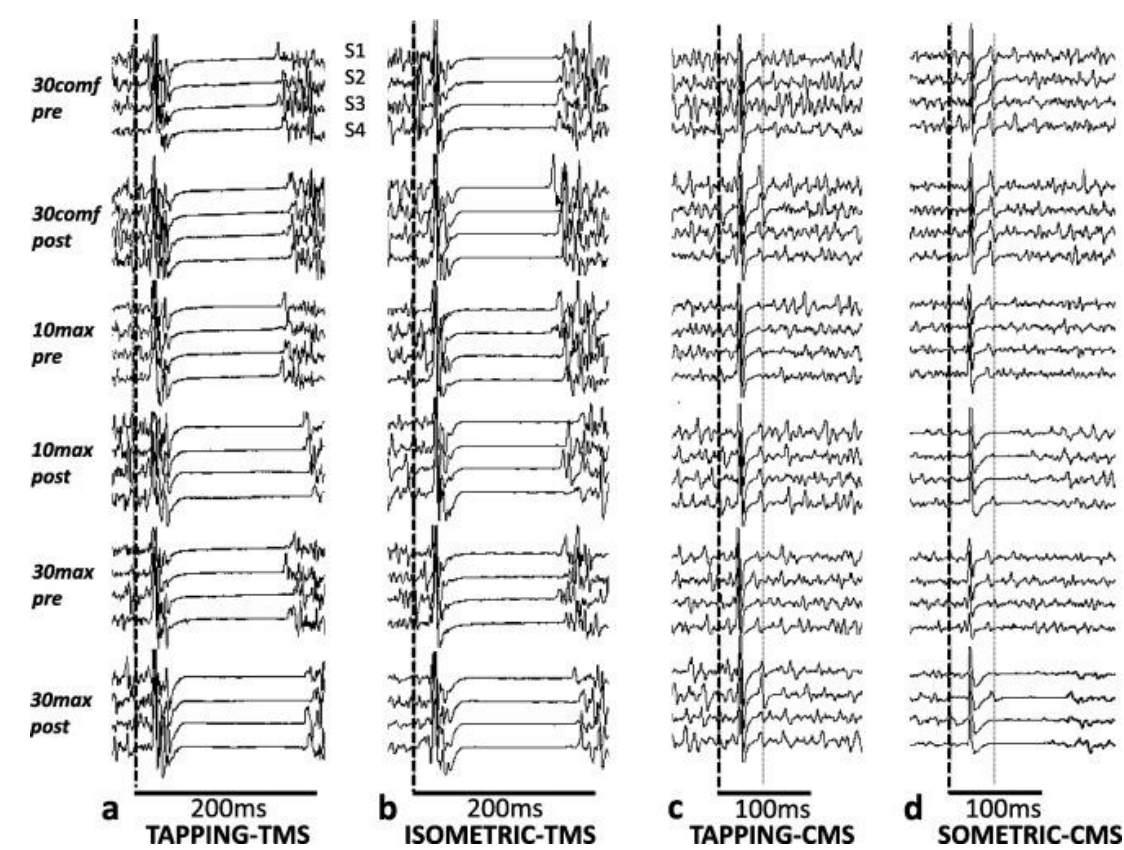

Fig. 5. Individual's recordings of the SP pre and post execution. S1...S4 are the sequential recordings of the four sets for a given time of evaluation. TMS-SP increased at post after $f t$ (a) and after iso (b), only at maximal modes. Conversely CMS-SP was unchanged after $f t$ in all modes (c), but increased after the maximal modes for the iso task, see (d) section. The vertical thick dashed lines indicate the time of stimulation. The vertical thin dashed line lies at the end of the transcortical reflex potential, evoked by CMS. The representation occludes part of the motor evoked potentials, in order to optimize the representation of the SPs.
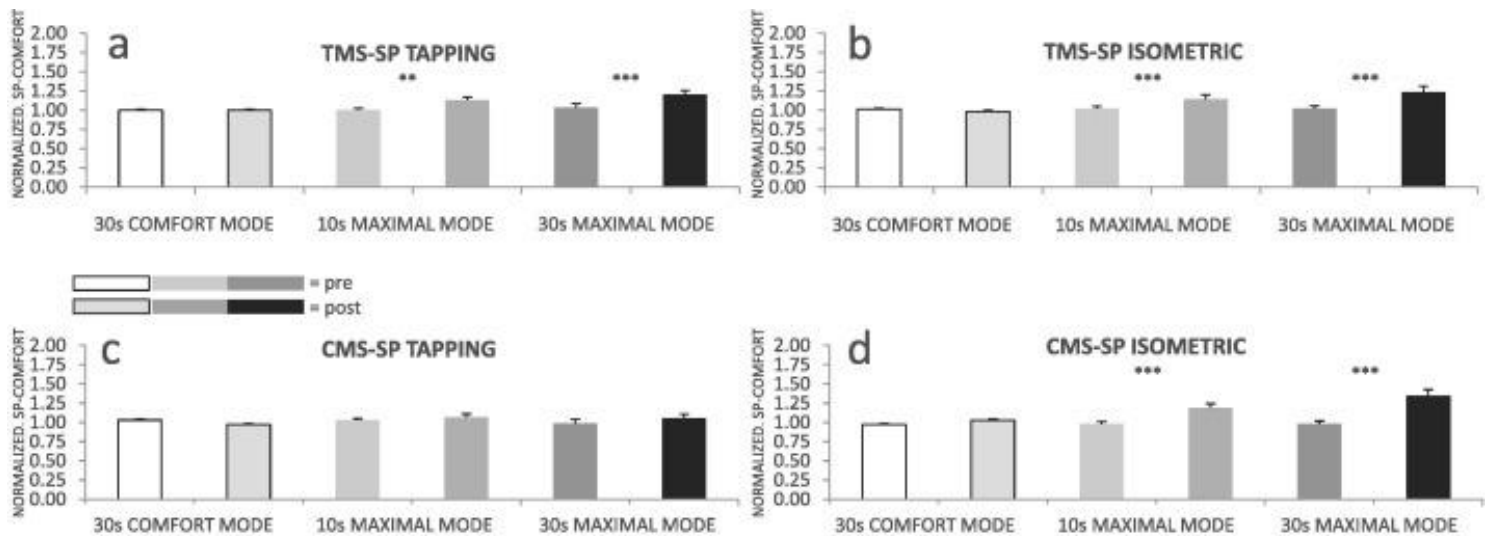

Fig. 6. SPs in response to TMS or CMS during fatiguing motor activities in all subjects. (a) and (b) show that the TMS-SP significantly increased after executing at maximal modes, either 10 or $30 \mathrm{~s}$ for $f t$ and iso. (c) However in no case did the CMS-SP increase after ft. (d) CMS-SP significantly increased after 10max and 30max iso. A significant effect of set never appeared for SP durations, therefore the four sets are shown pooled. The $y$-axis expresses the mean duration of the SP in comfort mode (pooling all sets and pre and post values).

For $f t$-task, the SP increase at post was significantly different for the CMS and TMS groups $\left(F_{1,19}=11.0, \boldsymbol{p}<\mathbf{0 . 0 1}_{\text {GROUP } \times \text { TIME }}\right)$. Subsequently, the analyses of the TMS-SPs showed a significant increase at the end of execution, which was also different for the execution modes $\left(F_{2,16}=9.1\right.$, $\left.\boldsymbol{p}<\mathbf{0 . 0 1}_{\mathrm{MODE}} \times \mathrm{TIME}\right)$. We observed that the increase of the TMS-SP was only significant after 10max $(\boldsymbol{p}<\mathbf{0 . 0 1}$ post hoc) and 30max $(\boldsymbol{p}<\mathbf{0 . 0 0 1}$ post hoc), not for comfort. Conversely the CMS-SP never increased at the end of the $f t\left(F_{1,11}=0.7, \boldsymbol{p}>\mathbf{0 . 0 5}\right.$ TIME $)$.

For iso-tasks the responses to fatigue of the SP induced by TMS and CMS were never significantly different. For both types of stimulation, the normalized SP increase at the end of the execution was 
different in the three modes $\left(F_{2,38}=25.0, \boldsymbol{p}<\mathbf{0 . 0 0 1}_{\mathrm{MODE}} \times \mathrm{TIME}\right)$; and the analyses by modes of execution indicated that for both the TMS and CMS groups, the SP increased significantly only after 10max and $30 \max$ (Post-hoc; $\boldsymbol{p}<\mathbf{0 . 0 0 1}$, in two cases) (Fig. 5b, d).

Set sequence never had a significant effect on SP $(\boldsymbol{p}>\mathbf{0 . 0 5}$ for main effects or interaction; i.e., in all modes and task).

\section{Discussion}

The main goal of this study was to determine the contribution of some spinal and supraspinal motor circuits to the generation of fatigue during short-lasting repetitive movements (finger tapping), if performed at the fastest possible rate. The site of CF was assessed by means of SPs evoked by TMS and electrical CMS at the time of fatigue within the FDI, a muscle with a more specific role in the tasks of our protocol than the other muscles explored in this work. Then during fatigue protocols, we evaluate SPs allowing no recovery of the neural system after the fatiguing activity (Taylor et al., 2000). While the SP generated by TMS has cortical and spinal components, the one generated by CMS has a purely spinal origin (Fuhr et al., 1991, Inghilleri et al., 1993 and Brasil-Neto et al., 1995). To our knowledge, this is the first study attributing fatigue induced by short-lasting repetitive $f t$ activity at the maximal rate to supraspinal structures, while controlling fast forms of fatigue-recovery from the task. Moreover, our results are consistent with a prominent contribution of spinal circuits to CF during isometric MVC ( Butler et al., 2003 and Klass et al., 2008), which does not rule out the additional contribution of a supraspinal component ( Gandevia et al., 1996, Di Lazzaro et al., 2003, Maruyama et al., 2006, Taylor and Gandevia, 2008 and McNeil et al., 2009).

The decrement in motor output was expressed as a reduction in $f t$ frequency, and in force for iso, in both cases at the end of 10max and 30max; this effect was observed in the two groups of subjects (one group stimulated with TMS, and the other with CMS), without significant differences in their task executions. We consider these changes in motor output as signs of fatigue, as they paralleled known fatigue behavioral markers like the reduction in MVC output right at the end (in continuation) of the fatiguing-tasks. There was also an increase in SP-durations evoked by TMS in both tasks after maximal modes (in a same group of subjects) ( Gandevia, 2001 and Taylor and Gandevia, 2001). Remarkably, CMS-SP (tested in a same group of subjects during $f t$ and iso tasks) increased only after maximal iso tasks, and not after $f t$ tasks; this is a clear sign of different contribution of spinal cord mechanisms to the development of fatigue during maximal iso and $f t$.

For fatigue-induced SP increase, there seems to be a ceiling effect at about $30 \mathrm{~s}$ (Taylor et al., 1996 and Taylor and Gandevia, 2001). In line with this, we never detected significant effects of set on SP, although it was present in behavior (MVC) in the case of iso.

The analyses of the SP-durations contribute to the understanding of the different origin of fatigue and task dependency (Enoka and Stuart, 1992) for $f t$ and iso at maximal modes. SP to TMS increased immediately at the end of both maximal $f t$ and iso. However, when in a same group of subjects we evaluated the task-dependency response of SP to CMS, it only increased after maximal iso activity, and not after maximal $f t$ activity, despite the fact that in these subjects maximal $f t$ induced reduction of tapping rate and MVC at the end of the task. CMS-SP emerges from spinal inhibitory mechanisms following motoneuronal excitation, such as after-hyperpolarization and recurrent inhibition ( Inghilleri et al., 1993 and Brasil-Neto et al., 1995), whereas the late part of the TMS-SP arises from the activation of inhibitory cortical interneurons projecting to the pyramidal cortico-spinal neurons (Inghilleri et al., 1993), acting via GABAb receptors ( Ziemann et al., 1993 and Ziemann, 2013). This suggests that fatigue induced by $f t$ in our study is not induced by the spinal mechanisms mentioned above, but takes place at supraspinal levels. On the other hand a spinal origin of fatigue seems to be clear for iso maximal ( Duchateau and Hainaut, 1993, Gandevia et al., 1999, Taylor et al., 1999, Butler et al., 2003 and McNeil et al., 2009), but likely to be present also at a supraspinal level (Di Lazzaro et al., 2003).

\section{Maximal voluntary contractions at fatigue time}

At the time of testing, our subjects exhibited a reduction of MVC as an expression of fatigue (Bigland-Ritchie and Woods, 1984 and Gandevia, 2001). The $f t$ and iso tasks were matched for efforts (comfort or maximal) and durations (10-30 s). However, it was observed that the different combinations of efforts, durations, and tasks had different impacts on the MVC at the time of fatigue testing, reflecting the fatigue tasks dependencies and suggesting different mechanisms of fatigue. For this reason, it is work for the future to design a protocol to match the drop of MVC after the different tasks (note that this was present in some sets of our protocol). 


\section{Fatigue accumulation after iso maximal tasks}

For iso tasks we observed an accumulation of fatigue set after set expressed in the MVC dropping of force, which was not different for subjects belonging to TMS or CMS groups. However, this effect was not reflected in a set effect for SPs (regardless TMS or CMS); this is in agreement with a previously described ceiling-effect for this variable ( Taylor et al., 1996 and Taylor and Gandevia, 2001). It is possible that some other circuits different to the ones explored in this study, or even peripheral expressions of fatigue, might explain this effect. A sign of peripheral fatigue, such the reduction on the CMAP (a marker of action potential sarcoplasmic propagation), should not be expectable for $30 \mathrm{~s}$ of maximal iso accordingly to previous studies ( Butler et al., 2003); but the effect of set after set accumulation on the CMAP response is unknown. Admittedly, the objective of this study (SP exploration) does not permit the recording of CMAP at the same time of testing. Notwithstanding that, although the CMAP is essential to normalized motor evoked potential amplitudes in fatiguing conditions, it does not condition the SP durations explained in this study.

\section{MVC drop during comfort mode evaluation at the time of fatigue}

MVC testing right at the end of the tasks (post) showed that the force dropped in all cases, including comfort modes for $f t$ and iso tasks. This suggests fatigue development even after comfort execution. However, the SP durations were not modified at post for comfort modes. The presence of fatigue after $f t$ comfort execution has been suggested previously ( Teo et al., 2012), but the evaluation was done with the fatigued muscle at rest and using TMS paired-pulse protocols. It is possible that fatigue after $f t$ and iso comfort develops first in cortical circuits un-explored in our protocol. For instance, intracortical inhibitory gabaergic mechanisms acting via GABAa receptors ( Werhahn et al., 1999, Di Lazzaro et al., 2000, Hallett, 2007 and Ziemann, 2013), or excitatory glutamatergic circuits responsible for motor evoked potential amplitude. In fact, an involvement of intracortical circuits (GABAa receptor mediated) has been suggested previously for the development of fatigue in $f t$ tasks ( Teo et al., 2012).

\section{Fatigue expressions on rate but not on amplitude for maximal ft tasks}

The subjects were asked to tap "as fast as possible", with no instructions about angular movement amplitude. In our study, fatigue never induced changes in angular amplitude during the task, and the movement amplitude did not change across execution modes (Rodrigues et al., 2009). This supports the view that the expression of supraspinal fatigue observed during maximal $f t$ was directly related to the tapping-rate, i.e. to the number of changes in the finger directions (sequences) per unit of time, and not to their amplitude. Interestingly, we observed that the level of activation of the FDI during maximal rate $f t$ is much lower than during iso MVC. This reinforces the view that the control of alternating fast sequences is demanding for supraspinal centers even when the level of muscle activation is not very high.

\section{Conclusion}

In conclusion, early forms of fatigue during short-lasting repetitive movements at the fastest rate do not originate at the circuits responsible for CMS-SP generation at spinal level. In contrast, short-lasting isometric MVC tasks induce spinal inhibitory mechanisms following motoneuronal excitation, likely explained by recurrent inhibition and after-hyperpolarization in response to the sustained maximal contraction. The fatigue induced by fast-rate repetitive movements possibly involves intracortical inhibitory circuits operating via GABAb interneurons, and its main expression is the decrease in tapping frequency.

Fatigue is one of the main signs in diseases of the spinal cord and supraspinal centers. We have provided neurophysiological evidence for different physiological mechanisms in $f t$ and iso. Our results, if replicated in the future with larger samples, may help to improve the design of the clinical tests aiming at the evaluation of CF in pathological and physiological conditions.

\section{Disclosure}

The authors declare no conflict of interests. 


\section{Authors contributions}

1. Conception and design of the experiments: P.A., V.R.G., J.C.

2. Collection, analysis and interpretation of data: P.A., V.R.G., Y.C.B., A.M., N.E., J.V., K.G., A.O., J.C.

3. Drafting the article or revising it critically for important intellectual content: P.A., V.R.G., Y.C.B., A.M., N.E., J.V., K.G., A.O., J.C.

\section{Acknowledgments}

Grants: This work was supported by Xunta de Galicia (Consellería de Educación 2007/000140-0; Ayudas Grupos Consolidados, Consellería de Educación, 2014 and Dirección Xeral de I+D+i; 20102012), Spain. V.R.G. and Y.C.B. are granted by the FPU-MECDAP2010-2774 and AP2010-2775 Spain.

\section{References}

Allen et al., 2008 D.G. Allen, G.D. Lamb, H. Westerblad Skeletal muscle fatigue: cellular mechanisms Physiol Rev, 88 (2008), pp. 287-332

Arias et al., 2012 P. Arias, V. Robles-Garcia, N. Espinosa, Y. Corral, J. Cudeiro Validity of the finger tapping test in Parkinson's disease, elderly and young healthy subjects: is there a role for central fatigue? Clin Neurophysiol, 123 (2012), pp. 2034-2041

Bigland-Ritchie and Woods, 1984 B. Bigland-Ritchie, J.J. Woods Changes in muscle contractile properties and neural control during human muscular fatigue Muscle Nerve, 7 (1984), pp. 691-699

Brasil-Neto et al., 1993 J.P. Brasil-Neto, A. Pascual-Leone, J. Valls-Sole, A. Cammarota, L.G. Cohen, M. Hallett Postexercise depression of motor evoked potentials: a measure of central nervous system fatigue Exp Brain Res, 93 (1993), pp. 181-184

Brasil-Neto et al., 1995 J.P. Brasil-Neto, A. Cammarota, J. Valls-Sole, A. Pascual-Leone, M. Hallett, L.G. Cohen Role of intracortical mechanisms in the late part of the silent period to transcranial stimulation of the human motor cortex Acta Neurol Scand, 92 (1995), pp. 383-386

Butler et al., 2003 J.E. Butler, J.L. Taylor, S.C. Gandevia Responses of human motoneurons to corticospinal stimulation during maximal voluntary contractions and ischemia J Neurosci, 23 (2003), pp. 10224-10230

Butler et al., 2012 J.E. Butler, N.C. Petersen, R.D. Herbert, S.C. Gandevia, J.L. Taylor Origin of the low-level EMG during the silent period following transcranial magnetic stimulation Clin Neurophysiol, 123 (2012), pp. 14091414

Cantello et al., 1992 R. Cantello, M. Gianelli, C. Civardi, R. Mutani Magnetic brain stimulation: the silent period after the motor evoked potential Neurology, 42 (1992), pp. 1951-1959

Di Lazzaro et al., 2000 V. Di Lazzaro, A. Oliviero, M. Meglio, B. Cioni, G. Tamburrini, P. Tonali, J.C. Rothwell Direct demonstration of the effect of lorazepam on the excitability of the human motor cortex Clin Neurophysiol, 111 (2000), pp. 794-799

Di Lazzaro et al., 2003 V. Di Lazzaro, A. Oliviero, P.A. Tonali, P. Mazzone, A. Insola, F. Pilato, E. Saturno, M. Dileone, J.C. Rothwell Direct demonstration of reduction of the output of the human motor cortex induced by a fatiguing muscle contraction Exp Brain Res, 149 (2003), pp. 535-538

Duchateau and Hainaut, 1993 J. Duchateau, K. Hainaut Behaviour of short and long latency reflexes in fatigued human muscles J Physiol, 471 (1993), pp. 787-799

Enoka and Stuart, 1992 R.M. Enoka, D.G. Stuart Neurobiology of muscle fatigue J Appl Physiol, 72 (1992), pp. $1631-1648$

Enoka and Duchateau, 2008 R.M. Enoka, J. Duchateau Muscle fatigue: what, why and how it influences muscle function J Physiol, 586 (2008), pp. 11-23

Enoka et al., 2011 R.M. Enoka, S. Baudry, T. Rudroff, D. Farina, M. Klass, J. Duchateau Unraveling the neurophysiology of muscle fatigue J Electromyogr Kinesiol, 21 (2011), pp. 208-219

Fuhr et al., 1991 P. Fuhr, R. Agostino, M. Hallett Spinal motor neuron excitability during the silent period after cortical stimulation Electroencephalogr Clin Neurophysiol, 81 (1991), pp. 257-262

Gandevia, 2001 S.C. Gandevia Spinal and supraspinal factors in human muscle fatigue Physiol Rev, 81 (2001), pp. 1725-1789

Gandevia et al., 1996 S.C. Gandevia, G.M. Allen, J.E. Butler, J.L. Taylor Supraspinal factors in human muscle fatigue: evidence for suboptimal output from the motor cortex J Physiol, 490 (Pt 2) (1996), pp. 529-536

Gandevia et al., 1999 S.C. Gandevia, N. Petersen, J.E. Butler, J.L. Taylor Impaired response of human motoneurones to corticospinal stimulation after voluntary exercise J Physiol, 521 (Pt 3) (1999), pp. 749-759

Hallett, 2007 M. Hallett Transcranial magnetic stimulation: a primer Neuron, 55 (2007), pp. 187-199

Hilty et al., 2011a L. Hilty, L. Jancke, R. Luechinger, U. Boutellier, K. Lutz Limitation of physical performance in a muscle fatiguing handgrip exercise is mediated by thalamo-insular activity Hum Brain Mapp, 32 (2011), pp. $2151-2160$

Hilty et al., 2011b L. Hilty, K. Lutz, K. Maurer, T. Rodenkirch, C.M. Spengler, U. Boutellier, L. Jancke, M. Amann Spinal opioid receptor-sensitive muscle afferents contribute to the fatigue-induced increase in intracortical inhibition in healthy humans Exp Physiol, 96 (2011), pp. 505-517

Hultborn et al., 1979 H. Hultborn, E. Pierrot-Deseilligny, H. Wigstrom Recurrent inhibition and afterhyperpolarization following motoneuronal discharge in the cat J Physiol, 297 (1979), pp. 253-266 
Inghilleri et al., 1993 M. Inghilleri, A. Berardelli, G. Cruccu, M. Manfredi Silent period evoked by transcranial stimulation of the human cortex and cervicomedullary junction J Physiol, 466 (1993), pp. 521-534

Jancke et al., 1998 L. Jancke, K. Specht, S. Mirzazade, R. Loose, M. Himmelbach, K. Lutz, N.J. Shah A parametric analysis of the 'rate effect' in the sensorimotor cortex: a functional magnetic resonance imaging analysis in human subjects Neurosci Lett, 252 (1998), pp. 37-40

Klass et al., 2008 M. Klass, M. Levenez, R.M. Enoka, J. Duchateau Spinal mechanisms contribute to differences in the time to failure of submaximal J Neurophysiol, 99 (2008), pp. 1096-1104

Kluger et al., 2013 B.M. Kluger, L.B. Krupp, R.M. Enoka Fatigue and fatigability in neurologic illnesses: proposal for a unified taxonomy Neurology, 80 (2013), pp. 409-416

Lutz et al., 2005 K. Lutz, S. Koeneke, T. Wustenberg, L. Jancke Asymmetry of cortical activation during maximum and convenient tapping speed Neurosci Lett, 373 (2005), pp. 61-66

Maruyama et al., 2006 A. Maruyama, K. Matsunaga, N. Tanaka, J.C. Rothwell Muscle fatigue decreases shortinterval intracortical inhibition after exhaustive intermittent tasks Clin Neurophysiol, 117 (2006), pp. 864-870

McNeil et al., 2009 C.J. McNeil, P.G. Martin, S.C. Gandevia, J.L. Taylor The response to paired motor cortical stimuli is abolished at a spinal level during human muscle fatigue J Physiol, 587 (2009), pp. 5601-5612

Rodrigues et al., 2009 J.P. Rodrigues, F.L. Mastaglia, G.W. Thickbroom Rapid slowing of maximal finger movement rate: fatigue of central motor control? Exp Brain Res, 196 (2009), pp. 557-563

Rossini et al., 1994 P.M. Rossini, A.T. Barker, A. Berardelli, M.D. Caramia, G. Caruso, R.Q. Cracco, M.R. Dimitrijevic, M. Hallett, Y. Katayama, C.H. Lucking, et al. Non-invasive electrical and magnetic stimulation of the brain, spinal cord and roots: basic principles and procedures for routine clinical application. Report of an IFCN committee Electroencephalogr Clin Neurophysiol, 91 (1994), pp. 79-92

Shimoyama et al., 1990 I. Shimoyama, T. Ninchoji, K. Uemura The finger-tapping test. A quantitative analysis Arch Neurol, 47 (1990), pp. 681-684

Taylor and Gandevia, 2004 J.L. Taylor, S.C. Gandevia Noninvasive stimulation of the human corticospinal tract J Appl Physiol (1985), 96 (2004), pp. 1496-1503

Taylor and Gandevia, 2001 J.L. Taylor, S.C. Gandevia Transcranial magnetic stimulation and human muscle fatigue Muscle Nerve, 24 (2001), pp. 18-29

Taylor and Gandevia, 2008 J.L. Taylor, S.C. Gandevia A comparison of central aspects of fatigue in submaximal and maximal voluntary contractions J Appl Physiol, 104 (2008), pp. 542-550

Taylor et al., 1996 J.L. Taylor, J.E. Butler, G.M. Allen, S.C. Gandevia Changes in motor cortical excitability during human muscle fatigue J Physiol, 490 (Pt 2) (1996), pp. 519-528

Taylor et al., 1999 J.L. Taylor, J.E. Butler, S.C. Gandevia Altered responses of human elbow flexors to peripheralnerve and cortical stimulation during a sustained maximal voluntary contraction Exp Brain Res, 127 (1999), pp. $108-115$

Taylor et al., 2000 J.L. Taylor, G.M. Allen, J.E. Butler, S.C. Gandevia Supraspinal fatigue during intermittent maximal voluntary contractions of the human elbow flexors J Appl Physiol, 89 (2000), pp. 305-313

Teo et al., 2012 W.P. Teo, J.P. Rodrigues, F.L. Mastaglia, G.W. Thickbroom Post-exercise depression in corticomotor excitability after dynamic movement: a general property of fatiguing and non-fatiguing exercise Exp Brain Res, 216 (2012), pp. 41-49

Ugawa et al., 1991 Y. Ugawa, J.C. Rothwell, B.L. Day, P.D. Thompson, C.D. Marsden Percutaneous electrical stimulation of corticospinal pathways at the level of the pyramidal decussation in humans Ann Neurol, 29 (1991), pp. $418-427$

Werhahn et al., 1999 K.J. Werhahn, E. Kunesch, S. Noachtar, R. Benecke, J. Classen Differential effects on motorcortical inhibition induced by blockade of GABA uptake in humans J Physiol, 517 (Pt 2) (1999), pp. 591597

Ziemann, 2013 U. Ziemann Pharmaco-transcranial magnetic stimulation studies of motor excitability Handb Clin Neurol, 116 (2013), pp. 387-397

Ziemann et al., 1993 U. Ziemann, J. Netz, A. Szelenyi, V. Homberg Spinal and supraspinal mechanisms contribute to the silent period in the contracting soleus muscle after transcranial magnetic stimulation of human motor cortex Neurosci Lett, 156 (1993), pp. 167-171

Zwarts et al., 2008 M.J. Zwarts, G. Bleijenberg, B.G. van Engelen Clinical neurophysiology of fatigue Clin Neurophysiol, 119 (2008), pp. 2-10 